and clinical outcomes but it has not been investigated as a measure of outcomes in atrial fibrillation (AF) patients.

Methods We performed a post-hoc analysis of the AFFIRM trial including patients with available echo data. Patients were stratified based on gender-adjusted echo derived interventricular septal (IVS) thickness, relative wall thickness (RWT), gender-adjusted LV mass and type of LV remodelling (normal LV geometry, concentric hypertrophy, eccentric hypertrophy, and concentric remodelling). Cox proportional hazards models were used for multivariate analyses of time to death and time to ischaemic stroke.

Results Of 4060 patients recruited in AFFIRM, sufficient echo data were available in 2433 patients $(60 \%)$. Multivariate analysis showed that moderate-severe LV (IVS diastolic dimension $>1.2 \mathrm{~cm}$ for women, $>1.3 \mathrm{~cm}$ for men) was associated with all cause mortality (HR 1.45, 95\% CI 1.13 to $1.86, p=0.003$ ). Concentric LV hypertrophy was associated with the worst outcome (defined as RWT $>0.42$ and LV mass $>224 \mathrm{~g}$ for men or LV mass $>162 \mathrm{~g}$ for women) ( $p=0.008$ vs, normal geometry-defined as RWT $\leq 0.42$ and LV mass $\leq 224 \mathrm{~g}$ for men or LV mass $\leq 162 \mathrm{~g}$ for women). In a multivariate model, including established clinical, demographic and echo risk factors, moderate-severe LV hypertrophy assessed by IVS thickness was the strongest echo predictor of stroke (HR 2.2, 95\% CI 1.3 to $3.7, \mathrm{p}=0.002$ ).

Conclusion In the AFFIRM Trial, LV hypertrophy assessed by gender-adjusted IVS thickness is an important risk factor for ischaemic stroke in patients with AF. LV hypertrophy assessed by gender-adjusted IVS thickness is associated with increased all cause mortality in AF patients.

\section{SUCCESSFUL APPLICATION OF A NOVEL RESTITUTION GRADIENT BASED MARKER OF VENTRICULAR ARRHYTHMIA TO PATIENTS WITH NON-ISCHAEMIC CONDITIONS}

doi:10.1136/heartjnl-2012-301877b.55

${ }^{1} \mathrm{M}$ I Smith, ${ }^{2} \mathrm{M}$ B Nicolson, ${ }^{1} \mathrm{P}$ Brown, ${ }^{3} \mathrm{~J}$ Tuan, ${ }^{3} \mathrm{~A}$ J Sandilands, ${ }^{3} \mathrm{P}$ J Stafford, ${ }^{1} \mathrm{~F}$ S Schlindwein, ${ }^{3} \mathrm{G}$ P McCann, ${ }^{3} \mathrm{G} \mathrm{A} \mathrm{Ng} .{ }^{1}$ University of Leicester, Leicester, UK; ${ }^{2}$ Leicester Cardiovascular Biomedical Research Unit, Leicester, UK; ${ }^{3}$ University Hospitals Leicester NHS Trust, Leicester, UK

Background There are major limitations with Sudden Cardiac Death (SCD) risk assessment, especially in patients without ischaemic heart disease (IHD). Electrical restitution, that is,. the relationship between action potential duration (APD) and preceding diastolic intervals (DI), is regarded as key to the initiation of ventricular arrhythmias (VAs). We have developed a novel measure of APD restitution heterogeneity based on 12-lead ECG recordings: Regional Restitution Instability Index (R2I2), and shown it to be predictive of $\mathrm{VA} /$ death in patients with established myocardial infarcts. ${ }^{1}$ This data represents the first application of R2I2 to patients at risk of SCD with non-ischaemic aetiologies.

Method A blinded retrospective study of 57 patients without IHD [Cases], undergoing ventricular tachycardia stimulation studies as SCD risk stratification for ICD, and 29 patients with structurally normal hearts [Controls] undergoing electrophysiology studies for supraventricular arrhythmias. Cases consisted of: 33 patients with dilated cardiomyopathy, 12 Brugada syndrome, 4 non-compaction cardiomyopathy, 3 myotonic dystrophy, 2 arrhythmogenic right ventricular dysplasia, 1 hypertrophic cardiomyopathy and 1 cardiac sarcoidosis. During programmed stimulation, surrogates of APD and DI were obtained from a high resolution surface ECG recording. Restitution curves were plotted with gradients for each lead calculated using overlapping least-squares linear segments. ${ }^{2}$ APD restitution gradient heterogeneity was measured and quantified as R2I2 (mean of the SD of the residuals from the mean at each segment for each ECG lead).
Results R2I2 was significantly higher in Cases compared to Controls (mean \pm SEM: $0.98 \pm 0.04$ vs $0.63 \pm 0.04, p<0.001$ ) (scatter plot). Six Cases reached the endpoint of VA/death (mean follow-up 5.2 years). There was a non-significant trend towards higher R2I2 in patients experiencing VA/death $(1.12 \pm 0.10$ vs $0.96 \pm 0.05, p=0.27)$. The graph shows the mean cutaneous restitution curve for all leads and all patients, steeper curves with longer OTp (APD) were seen in the Case group.

Conclusion The R2I2 was higher in patients at risk of SCD than controls. Use of cutaneous surrogates to quantify APD restitution heterogeneity and assess risk of SCD shows promise in patients with non-ischaemic aetiologies.
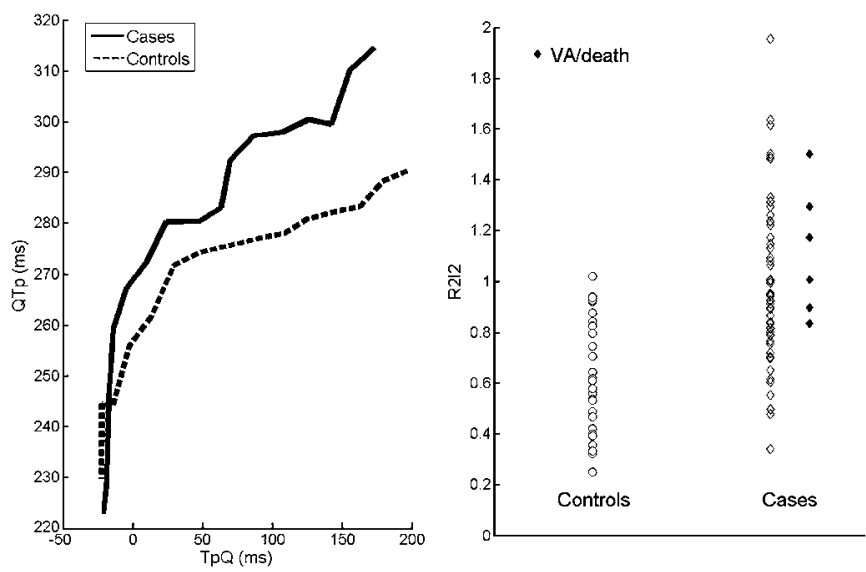

Abstract 055 Figure 1

\section{REFERENCES}

1. Nicolson WB, et al. Europace 2011;13(S4):iv1.

2. Taggart P, et al. Circulation 2003;107:285-9.

\section{LEUCOCYTE TELOMERE/TELOMERASE DYNAMICS IN PATIENTS WITH IMPLANTABLE CARDIOVERTER DEFIBRILLATOR; POTENTIAL BIOMARKER FOR VENTRICULAR ARRHYTHMIAS}

doi:10.1136/heartinl-2012-301877b.56

${ }^{1}$ V S Sawhney, ${ }^{* 1} \mathrm{~N}$ G C Campbell, ${ }^{2}$ S W B Brouilette, ${ }^{2} Y$ S Shintani, ${ }^{2}$ S R C Coppen, ${ }^{2} \mathrm{~T}$ N Narita, ${ }^{2} \mathrm{C}$ I lkebe, ${ }^{2} \mathrm{M}$ K Kaneko, ${ }^{2} \mathrm{~K}$ Y Yashiro, ${ }^{1} \mathrm{~V}$ B Baker, ${ }^{1} \mathrm{~F}$ G Goromonzi, ${ }^{1} \mathrm{M}$ A Abbott, ${ }^{1} \mathrm{E} D$ Duncan, ${ }^{1} \mathrm{M} D$ Dhinoja, ${ }^{1} \mathrm{M}$ J E Earley, ${ }^{1} \mathrm{~S} S$ Sporton, ${ }^{2} \mathrm{~K}$ S Suzuki, ${ }^{1} \mathrm{R}$ J S Schilling. ${ }^{1}$ St Bartholomew's Hospital, London, UK; ${ }^{2}$ William Harvey Research Institute, UK

Introduction Implantable cardioverter defibrillators (ICDs) have been shown to reduce mortality in patients with ischaemic cardiomyopathy at a high risk of ventricular arrhythmias (VA), which are the commonest cause of sudden death. However, ICDs are associated with morbidity and mortality Importantly $67 \%$ of patients never receive an appropriate shock after ICD implantation under the current indication, suggesting a need for better risk stratification tools. Telomere and telomerase in leucocytes have recently been shown to correlate with biological aging, health status, and also with pathogenesis/prognosis of various cardiovascular diseases. We hypothesise that the leucocyte telomere length and/or telomerase activity correlate with the incidence of VA in ischaemic cardiomyopathy patients.

Methods 73 ischaemic cardiomyopathy Caucasian patients with primary prevention ICDs were recruited to this retrospective study between October 2010 and January 2011 at St Bartholomew's Hospital. Concentrated leucocyte fraction was obtained from venous blood sample of recruited patients and stored at $-80^{\circ} \mathrm{C}$ in an 
anonymous manner. Genomic DNA was extracted from these and telomere length measured by telomere PCR. Each telomere length was expressed as a ratio (telomere length: single copy gene). Telomerase activity was measured using Roche Telo TAAGGG ELISA kit. All samples were analysed in duplicate and investigators were semiblinded to arrhythmia history. Continuous data were compared using unpaired $t$ test and categorical data by $\chi^{2}$ test. Logistic regression analysis was performed to determine if telomere length/ telomerase activity independently predict the likelihood of a shock (fatal VA). A probability value of $\mathrm{p}<0.05$ was defined as significant. Results There were no significant differences between the Shock (patients received appropriate shocks from ICD; $n=25$ ) and Nonshock (patients received no shock; $n=48$ ) groups in terms of baseline demographics as shown in Abstract 056 table 1. There was no significant difference in the age, sex and WCC adjusted telomere length between the Shock and Non-shock groups $(p=0.439)$. Expected age and WCC turnover related telomere attrition $(p=0.031$ and 0.031) was observed. In contrast, telomerase activity was significantly higher in the Shock group thank in the Non-shock group (0.5682 vs 0.2105$)$ and co-related to the incidence of shock $(p=0.01)$. This did not appear to be related to an acute response associated with VA.

\section{Abstract 056 Table 1}

\begin{tabular}{llll}
\hline & Group1 (shock) & Group 2 (non-shock) & p Value \\
\hline Number of patients & 25 & 48 & \\
Age, mean (range) & $70.8(56-87)$ & $70.8(46-87)$ & 0.97 \\
Female gender (\%) & 4 & 16.67 & 0.15 \\
Diabetes (\%) & $5(20)$ & $14(29.2)$ & 0.57 \\
Hypertension (\%) & $5(20)$ & $14(29.2)$ & 0.57 \\
Time since implant (months), mean & 50.68 & 34.13 & 0.06 \\
LVEF & 27.64 & 27.29 & 0.84 \\
\hline
\end{tabular}

Conclusion This is the first study to characterise the telomere dynamics of patients at high risk of sudden cardiac death and corelate this with the incidence of VA. Leucocyte telomerase activity independently predicted the likelihood of shock in ischaemic cardiomyopathy patients with primary prevention ICDs. Thus leucocyte telomerase activity may be a potential biomarker for prediction of fatal arrhythmia and guide ICD prescription. To validate these results, a prospective study is now ongoing.

\section{A RANDOMISED CONTROLLED TRIAL OF CATHETER ABLATION VS MEDICAL TREATMENT OF ATRIAL FIBRILLATION IN HEART FAILURE (THE CAMTAF TRIAL)}

doi:10.1136/heartjnl-2012-301877b.57

${ }^{1} \mathrm{R}$ J Hunter, ${ }^{1} \mathrm{~T}$ J Berriman, ${ }^{1} \mathrm{I}$ Diab, ${ }^{1} \mathrm{R}$ Kamdar, ${ }^{1} \mathrm{~L}$ Richmond, ${ }^{1} \mathrm{~V}$ Baker, ${ }^{1} \mathrm{~F}$ Goromonzi, ${ }^{1}$ E Duncan, ${ }^{1} \mathrm{~V}$ Sawhney, ${ }^{2} \mathrm{~B}$ Unsworth, ${ }^{2} \mathrm{~J}$ Mayet, ${ }^{1} \mathrm{M}$ Dhinoja, ${ }^{1} \mathrm{M} \mathrm{J}$ Earley, ${ }^{1} \mathrm{~S}$ Sporton, ${ }^{1} \mathrm{R}$ J Schilling. 'St Bartholomew's Hospital, London, UK; ${ }^{2}$ Internationl Centre for Circulatory Health, St Mary's Hospital, Imperial College London, London, UK

Introduction Although atrial fibrillation (AF) has deleterious effects in patients with heart failure (HF), rhythm control using medication has limited efficacy. Catheter ablation (CA) of AF is effective in restoring sinus rhythm, raising the question: if it can be shown to be safe and effective in HF patients, might it improve left ventricular (LV) function and HF symptoms? We sought to compare the impact of a CA strategy to a medical rate control strategy (MED) in patients with persistent AF and HF.

Methods Patients with persistent AF, symptomatic HF, and a LV ejection fraction $(\mathrm{EF})<50 \%$, were randomised to CA or MED. HF medication and anticoagulation were optimised prior to baseline observations. For those with recurrent AF in the CA group, a repeat procedure was performed at the end of the 3-month blanking period and follow-up re-started. The primary end-point was the difference in LV EF between groups on echocardiography at 6 months. Echocardiographic data were anonymised and core reported by a blinded collaborating centre. Secondary end-points included difference in NYHA class, Minnesota living with heart failure questionnaire score, and peak oxygen consumption at 6 months.

Results 55 patients were randomised, but five were excluded (LV function normalised during optimisation of medications prior to baseline tests in 2, and 3 withdrew un-happy with their treatment allocation). Patients were $58 \pm 11$ years and $96 \%$ were male. Baseline LV EF was $31 \pm 10 \%$ in the CA group and $33 \pm 9 \%$ in the MED group. NYHA class was $2.5 \pm 0.5$ in both groups. Patients underwent $1.6 \pm 0.7$ procedures. There were two complications: (1) stroke and 1 tamponade. In the CA group 1 patient withdrew after a procedural stroke, and in the MED group 1 patient died. In total 21 of 24 in the MED group and 24 of 26 in the CA group had reached 6-months follow-up and were included in this analysis of the primary endpoint. Freedom from AF was achieved in 21/24 (88\%) off antiarrhythmic drugs in the CA group, whereas all those in the MED group remained in AF. LV EF in the CA group at 6 months was $39 \pm 10 \%$ compared to $32 \pm 13 \%$ in the MED group $(p<0.05)$. NYHA class was also significantly lower in the CA group at 6 months $(1.7 \pm 0.8$ compared to $2.3 \pm 0.6$ in the medical group; $p<0.05)$. CA was associated with better peak oxygen consumption $(22.4 \pm 6.3 \mathrm{ml} /$ $\mathrm{kg} / \mathrm{min}$ vs $18.6 \pm 6.0 \mathrm{ml} / \mathrm{kg} / \mathrm{min}, \mathrm{p}=0.053)$ and Minnesota living with heart failure questionnaire score $(24 \pm 23$ vs $48 \pm 25, p=0.002)$ compared to the MED group.

Conclusions CA is effective in restoring sinus rhythm in the majority of patients with persistent AF. A CA strategy for patients with AF and HF is associated with improved LV function and heart failure symptoms compared to medical treatment alone.

\section{THE RELATIONSHIP OF LEFT ATRIAL REMODELLING TO ATRIAL FIBRILLATION BURDEN IN PACEMAKER POPULATION}

doi:10.1136/heartjnl-2012-301877b.58

C W Khoo, * S Krishnamoorthy, G Dwivedi, A Blann, H S Lim, G Y H Lip. University Department of Medicine Centre for Cardiovascular Sciences, City Hospital, Birmingham, UK

Background Cumulative ventricular pacing (VP) is associated with development of atrial fibrillation and cardiac remodelling. Contemporary pacemaker devices are able to quantify atrial highrate episodes (AHREs) and atrial fibrillation burden (AFB). No previous study has investigated the relationship of reverse LA remodelling and AHRE. We tested the hypothesis that reverse LA remodelling parameters are associated with greater AF burden and larger percentage of cumulative ventricular pacing in pacemaker population.

Methods 101 patients [mean age $72 \pm 11$ years, 69 (68\%) men), mean follow-up of $359.09 \pm 83.85$ days] with dual-chamber pacemaker underwent two-dimension (2D) and tissue Doppler imaging (TDI) echocardiography. LA volume (LAV) was evaluated by area-length method and function by septal A'. LV systolic and diastolic parameters were evaluated by mitral inflow velocity (E, A), LV ejection fraction (biplane Simpson's) and septal TDI velocity. The presence of AHREs (defined by atrial-rate $\geq 220 \mathrm{bpm}$ and $\geq 5 \mathrm{~min}$ ) and AFB were derived from pacemaker diagnostics.

Results The incidence of AHRE in our study was $35 \%(n=35)$ with median percentage AF burden of 2.6 (IOR 0.1-26.4). Patients with AHREs had significantly larger LA volume $(p=0.014)$ and reduced LA function at follow-up $(\mathrm{p}=0.034)$, as were LV diastolic parameters, LV and RV longitudinal systolic function (all $p<0.05$ ) [Abstract 058 